\title{
Estrategias de financiamiento a corto plazo aplicadas por el sector hotelero en el municipio Cabimas
}

\section{Short term financing strategies applied by the hotel sector in the municipality of Cabimas}

\author{
४ Sorrentino Emanuele \\ ema.sorcas@gmail.com \\ ORCID: 0000-0002-5458-1950 \\ Universidad de Carabobo. Venezuela \\ Artículo recibido en julio 2018 / Arbitrado en agosto 2018 / Publicado en enero 2019

En la investigación se identificaron las estrategias de financiamiento a corto plazo aplicadas por el sector hotelero en el municipio Cabimas. La metodología fue de tipo descriptiva, con diseño no experimental, de campo y transeccional. La población quedó conformada por los hoteles que hacen vida activa en el municipio Cabimas. Para la recolección de datos se empleó la encuesta mediante la aplicación de un cuestionario, contentivo de 18 ítems con escala tipo Lickert. La validez se realizó a través del juicio de cinco (5) expertos, y para calcular su confiabilidad se empleó el Coeficiente Alpha de Cronbach dando como resultado 0,94. El análisis de los datos se realizó mediante estadística descriptiva, con base en la media aritmética. Del análisis realizado se pudo evidenciar que estos hoteles poseen moderada aplicación del financiamiento de los socios, pasivos acumulados, crédito comercial, cuentas por pagar, línea de crédito y proveedores, delineándola como debilidades.

Palabras clave: Crédito comercial, cuentas por pagar, estrategias de financiamiento a corto plazo, financiamiento de los socios, línea de crédito, pasivos acumulados, proveedores

The research identified the short term financing strategies applied by the hotel sector in the Cabimas municipality. The methodology was descriptive, with a nonexperimental, field and transectional design. The population was made up of hotels that make active life in the Cabimas municipality. For data collection, the survey was used through the application of a questionnaire, containing 18 items with a Lickerttype scale. Validity was made through the judgment of five (5) experts, and Cronbach's Alpha Coefficient was used to calculate its reliability, resulting in 0.94. Data analysis was performed using descriptive statistics, based on the arithmetic mean. From the analysis carried out, it was evident that these hotels have moderate application of member financing, accumulated liabilities, commercial credit, accounts payable, line of credit and suppliers, outlining it as weaknesses.

Keywords: Commercial credit, accounts payable, short term financing strategies, partner fntaning, ine of redit, accumulated liabilities, suppliers 
Al observar el mundo competitivo en el cual se encuentran inmersas las empresas en la actualidad, se hace necesario la adaptación al entorno, para ello los gerentes, además de asumir las responsabilidades que les corresponden para enfrentar al proceso de globalización, también deben considerar el rol a cumplir en ellas, para reafirmar con efectividad su desempeño como respuesta a las exigencias de este contexto turbulento y lleno de cambios de paradigmas.

Por ello, al adentrarse en el siglo XXI es necesario que los gerentes de las empresas actuales, deban asumir responsabilidades correspondientes para enfrentar el proceso de globalización, indistintamente del sector económico donde se desenvuelva. Esto constituye un desafío para los países de Latinoamérica, al tener que adaptarse a un ambiente de negocio abierto, con orientación internacional, conservando siempre su cultura, independencia, tradiciones, es decir sus raíces, donde las estrategias y políticas deberían incrementar la competitividad.

En este orden de ideas, el dinamismo experimentado en el orden económico mundial, producto de la globalización, ha propiciado la orientación estratégica en las empresas independientemente de su naturaleza y razón social, dirigida en lograr el eficaz uso de los recursos, fomentando la realización de esfuerzos en virtud de alcanzar niveles óptimos en las actividades productivas; en otras palabras, dirigir de manera eficaz y eficiente la gestión funcional, a los fines de lograr un liderazgo empresarial en su entorno.

Así entonces, la manera eficiente de armonizar el uso de recursos humanos, materiales, técnicos, de tiempo y financieros; inciden en el desarrollo de sus procesos, tanto administrativos como operativos, de igual modo, en la calidad de servicios que ofrecen dichas organizaciones, por tanto, es necesario el conocimiento concreto de tareas a realizar en los departamentos que conforman las organizaciones.

Así mismo, García y Paredes (2014) refieren, las organizaciones cuya razón social es prestar servicios, requieren de herramientas propiciadoras de competitividad, siendo las estrategias de financiamiento elementos que permiten incorporar diversos ciclos o etapas de procesos clave, impulsando operaciones que desarrollen de manera continua, armónica y orientada al éxito.

En este marco, Sánchez (2013) explica, el desempeño de estrategias de financiamiento tanto a corto como a largo plazo, les permiten a las empresas lograr el normal y eficaz desarrollo de sus actividades administrativas y operativas; en ese sentido, dichas estrategias han de ser efectivas, contributivas para alcanzar el objetivo de reducir los costos de financiamiento, sobre todo colaborar a mejorar la gestión global de estas organizaciones. Así, la función de las estrategias de financiamiento es proporcionar recursos requeridos por las empresas en condiciones favorables para ellas. Por tanto el concepto de financiamiento hace resaltar la esencia de la función financiera que consiste en mantener al negocio con el capital necesario para lograr sus metas.

Al respecto, Gómez (2013) afirma, las estrategias de financiamiento constituyen uno de los elementos que determinan la necesidad de establecer correctivos y controles acerca de las políticas de gastos operativos de las empresas, pues permiten ajustarlos a las circunstancias. Mientras, García y Paredes (2014) refieren, estas estrategias permiten la adquisición de recursos para obtener bienes tangibles y elementos monetarios necesarios para mejorar la gestión funcional de la empresa 
integrando sus operaciones y permitiendo su conformidad hasta que la empresa se sostenga por sí misma.

Antes estas consideraciones, para Gutiérrez (2010), la aplicación eficaz y eficiente de las estrategias de financiamiento es relevante para las organizaciones, sobre todo en empresas de servicios, por ser una de las fuentes generadoras de ingresos que les permite cumplir compromisos con terceras personas, lograr la realización de sus inversiones, las cuales deben ser controladas efectivamente. Para ello, las empresas deben identificar el costo financiero asociado.

Ahora bien, una fuente de financiamiento no es más que, fondos o recursos financieros que procura la empresa para financiar total o parcial sus objetivos financieros. La selección de la fuente de financiamiento adecuada, permitiría no solo disminuir el riesgo comercial y financiero del negocio, sino también permitiría maximizar el valor de las acciones, objetivo financiero de toda organización.

Sin embargo, en Venezuela, en los últimos años, tanto la empresa pública como la privada, han tenido grandes inconvenientes de diferente índole para su desarrollo, tales como: políticos (inestabilidad, incertidumbre), económicos (inflación, altas tasas de interés, devaluación) y legales (reformas de las distintas leyes), lo que ha traído como consecuencia altos niveles de capacidad ociosa y limitado grado de abastecimiento y competencia, originando una baja productividad y competitividad, según lo señalan Oldenburg y otros (2014) citando a Chillida (2005).

Hoy en día la administración financiera de las empresas venezolanas, se enfrentan a unos de sus más grandes retos, decidir, iqué fuente de financiamiento elegir ante la situación económica que vive el país que les afecta directamente en sus decisiones financieras? Dicha situación constituye una fuente de conflictos entre los diferentes niveles organizacionales comprometidos en la gestión de dichas empresas.

Adicional a esto, la mayoría de las empresas venezolanas presentan limitaciones en la gestión financiera estratégica conducente a generar los cursos de acciones que permitan mejorar, de una forma efectiva y eficaz, los procesos en la administración financiera de cada organización, tal como lo indican Oldenburg y otros (2014) citando a Chillida (2005).

En la actualidad, varios de los hoteles en el municipio Cabimas se enfrentan a una serie de problemas vinculados al desarrollo de sus estrategias de financiamiento, entre las que destacan la selección de fuentes de financiamiento adecuadas y la aplicación de estrategias de financiamiento bien definidas para el logro de sus objetivos, con el fin de obtener de manera continua, insumos, bienes de capital (intermedios y terminados) en condiciones favorables de calidad y precio, asegurando el éxito económico y su permanencia en el mercado.

Ahora bien, según la información aportada por los gerentes de estos hoteles, en los actuales momentos, para la permanencia en el mercado de estas organizaciones, existe la necesidad de establecer estrategias de financiamiento, tanto a corto como a largo plazo, como uno de los requerimientos más inmediatos.

Aunado a esta situación, el financiamiento en estas empresas, como toda empresa, está vinculado a fondos propios y al sistema financiero general. Cuando las empresas se plantean la necesidad de obtener nuevos medios de financiamiento deben primero revisar si internamente se poseen estos medios, 
y si son utilizables sin arriesgar su normal funcionamiento, en caso de no poder resolver internamente dicho financiamiento la empresa acudirá al mercado de capitales para resolver sus problemas de captación de recursos financieros a través del financiamiento externo.

De manera específica, el investigador pudo conocer, en estas entrevistas informales, como en la mayoría de los hoteles se padece de una ausencia marcada de mecanismos estratégicos para lograr financiamiento, el cual podría estar dirigido a la adquisición de equipos ofimáticos con programas actualizados en el área financiera que permita tener oportunamente información de los inventarios, nómina de personal, contrataciones eventuales. Asimismo, se puntualizaba un bajo nivel de incentivos crediticios por parte del sector financiero al sector hotelero, a lo cual se le añade falta de capital por parte de los socios, un exceso de deudas para financiar las inversiones y un exceso de deudas a corto plazo; situaciones que justifican la búsqueda de estrategias de financiamiento a corto plazo.

En efecto, la inquietud principal de esta investigación es la necesidad de estrategias de financiamiento acordes a la problemática planteada que permitan avanzar y mantenerse en el mundo competitivo empresarial a los hoteles en estudio. La gerencia financiera de éstos debe contar con las herramientas necesarias para tomar decisiones oportunas que coadyuven a aumentar sus recursos.
Por lo antes expuesto, se hizo necesario realizar un estudio dirigido a identificar las estrategias de financiamiento a corto plazo aplicadas por el sector hotelero en el municipio Cabimas, para determinar los factores que incidían en la formulación de dichas estrategias.

\section{MATERIALES Y MÉTODO}

La metodología fue de tipo descriptiva, con diseño no experimental, de campo y transeccional. La población quedó conformada por los hoteles que hacen vida activa en el municipio Cabimas y que cumplían con ciertos requisitos de inclusión. Bajo el cumplimiento de estos criterios se identificaron cuatro (4) hoteles: Hotel Cabimas Internacional C.A.; Hotel Costa Sol C.A.; Hotel D`Maio C.A y Hotel Buena Vista C.A. Los datos fueron recolectados de una población finita conformada por gerentes generales, gerentes de finanzas y gerentes administrativos, con un total de 12 sujetos informantes.

Para la recolección de datos se empleó la encuesta mediante la aplicación de un cuestionario, contentivo de 18 ítems con escala tipo Lickert. La validez se realizó a través del juicio de cinco (5) expertos, y para calcular su confiabilidad se empleó el Coeficiente Alpha de Cronbach dando como resultado 0,94 considerado de alta confiabilidad. El análisis de los datos se realizó mediante estadística descriptiva, con base en la media aritmética, para lo cual se diseñó el baremo pertinente, mostrado en la Tabla 1. 
Tabla l. Baremo para la interpretación de la media aritmética

\begin{tabular}{|c|c|c|}
\hline RANGO & CATEGORÍA & DESCRIPCIÓN \\
\hline $4,21-5,00$ & Muy alta aplicación & Si la aplicación es muy alta o alta se considera una \\
\hline $3,41-4,20$ & Alta aplicación & $\begin{array}{l}\text { fortaleza para el sector hotelero, en cuanto al manejo de } \\
\text { sus estrategias de financiamiento }\end{array}$ \\
\hline $2,61-3,40$ & Moderada aplicación & \\
\hline $1,81-2,60$ & Baja aplicación & debilidad para el sector hotelero, en cuanto al manejo de \\
\hline $1,00-1,80$ & Muy baja aplicación & sus estrategias de financiamiento \\
\hline
\end{tabular}

Fuente: Elaboración propia (2020)

\section{RESULTADOS Y DISCUSIÓN}

Tras la aplicación del cuestionario, se logró obtener los siguientes resultados: en la tabla 2, se muestra una media para la dimensión estrategias de financiamiento a corto plazo de 3,33 , calificando con la categoría de moderada aplicación a las estrategias aplicadas por el sector hotelero del municipio Cabimas, considerándola como una debilidad para este sector en cuanto al manejo de sus estrategias de financiamiento. Al detalle, las dos (2) subdimensiones medidas muestran moderada aplicación, tanto las estrategias de financiamiento internas a corto plazo $(2,83)$ como las externas a corto plazo $(3,83)$, tal como lo reflejan las medias alcanzadas por dichas sub-dimensiones, e interpretándose de acuerdo al baremo diseñado para tal fin.

Tabla 2. Dimensión: Estrategias de financiamiento a corto plazo

\begin{tabular}{lcc}
\hline SUB-DIMENSIONES & MEDIA & CATEGORÍA \\
\hline Fuentes internas a corto plazo & 2,83 & Moderada aplicación/debilidad \\
Fuentes externas a corto plazo & 3,83 & Moderada aplicación/debilidad \\
DIMENSIÓN & 3,33 & Moderada aplicación/debilidad \\
\hline
\end{tabular}

Fuente: Elaboración propia (2020)

Este resultado, de moderada aplicación, no logra validar lo expuesto por Morales y Morales (2014) cuando afirman, la estrategia para el financiamiento que adopte una empresa en el corto plazo deberá considerar el origen del financiamiento, si será de fuentes internas o externas, o una combinación de ambas.

De igual manera, se evidencia poca congruencia con García y Paredes (2014) quienes refieren, la estrategia de financiamiento a corto plazo considera fuentes internas y externas a la empresa, definidas por las instituciones nacionales (públicas y privadas) e internacionales que otorgan recursos económicos. Al mismo tiempo, logran mediana coincidencia con el investigador cuando asevera, al momento de elegir entre las diferentes alternativas de fuentes de financiamiento, el estratega financiero del sector hotelero del municipio Cabimas, deberá considerar el origen de los recursos tanto internos como externos. 
Continuando con el análisis, al desglose de cada una de las sub-dimensiones de esta dimensión a continuación se especifican los resultados obtenidos correspondientes a la primera de ellas, denominada fuentes internas a corto plazo, mostrados en la tabla 3 donde se recoge el resultado de la sub-dimensión y cada uno de sus indicadores. Como se observa en la tabla, las fuentes internas de a corto plazo se posicionan en moderada aplicación, siendo esto una debilidad para este sector en el manejo de sus estrategias de financiamiento a corto plazo.

Tabla 3. Sub-Dimensión: Fuentes internas a corto plazo

\begin{tabular}{lll}
\hline INDICADORES & MEDIA & CATEGORÍA \\
\hline Financiamiento de los socios & 3,44 & Alta aplicación/fortaleza \\
Pasivos acumulados & 1,89 & Baja aplicación/debilidad \\
Crédito comercial & 3,39 & Moderada aplicación/debilidad \\
Cuentas por pagar & 2,58 & Baja aplicación/debilidad \\
SUB - DIMENSIÓN & 2,83 & Moderada aplicación/debilidad \\
\hline
\end{tabular}

Fuente: Elaboración propia (2020)

La situación reseñada, es producto del comportamiento reflejado por los indicadores utilizados para medir esta sub-dimensión donde se alcanzan diferentes niveles de aplicación de las fuentes internas a corto plazo. En detalle, se evidencia alta aplicación de la fuente interna basada en el financiamiento a los socios cuya media de 3,44 así lo ratifica, convirtiendo la aplicación de esta fuente en una fortaleza para el sector estudiado. Ahora bien, otorgan mediana aplicación a las fuentes internas relacionadas a al crédito comercial, mostrada por una media de 3,39 siendo debilidad para el sector según el baremo utilizado.

No obstante, catalogan con la categoría de baja aplicación lo referido a las fuentes internas provenientes de los pasivos acumulados y las cuentas por pagar, como lo determinan sus medias de 1,89 y 2,58, respectivamente, viéndolas como debilidades en la aplicación de sus estrategias de financiamiento a corto plazo de origen interno.

Lo descrito, en estos resultados, alcanza validez con la postura de Mora (2017) quien plantea, la financiación interna o propia, es el dinero aportado por propietarios y forma lo denominado capital o más concretamente capital propio la cual posee las siguientes características: la cesión de capital es por tiempo ilimitado, da derecho a la propiedad de la empresa, proporciona el derecho a los beneficios de la empresa, esto en cuanto a la alta aplicación del financiamiento de los socios.

Sin embargo, se logra poca congruencia con lo asumido por Morales y Morales (2014) cuando afirman, la estrategia para el financiamiento que adopte la empresa en el corto plazo deberá considerar el origen del financiamiento, si este es de fuentes internas entonces provienen del financiamiento de los 
socios, pasivos acumulados, crédito comercial, cuentas por pagar, emisión de nuevas acciones e ingreso de nuevos socios.

Al respecto, el investigador infiere, en el sector hotelero se da mayor aplicación a la estrategia interna soportada en el financiamiento de los socios, no así al resto de las opciones consideradas en esta investigación: crédito comercial pasivos acumulados y las cuentas por pagar. Validando lo descrito por el investigador en las bases teóricas, el financiamiento interno constituye la manera en la cual una empresa puede captar los fondos o recursos financieros para llevar a cabo sus metas de crecimiento y progreso, de esta forma, puede entenderse como una fuente muy ventajosa para la empresa debido a su bajo costo, pues estos fondos provienen de sus socios, lo cual pareciera ideal como una fuente de financiamiento para el sector hotelero del municipio Cabimas.

De esta manera, analizando minuciosamente el comportamiento de cada uno de los indicadores de la sub-dimensión analizada, referente a lo consultado en cada ítem se obtuvo, en cuanto al financiamiento de los socios, con muy alta aplicación $(4,25)$, en los hoteles bajo estudio, cuando se proponen realizar aumentos del capital social acuden a las aportaciones de capital de los mismos accionistas; y con alta aplicación $(4,17)$ son utilizadas las aportaciones de los socios como fuente interna de financiamiento; convirtiendo estas actividades en fortalezas para el sector en el manejo de esta fuente interna a corto plazo.

Adicionalmente, se observa baja aplicación de todas las actividades medidas por los ítems en el indicador pasivos acumulados, siendo debilidades para el sector en la aplicación de sus estrategias de financiamiento a corto plazo, en cuanto a si utilizan los pasivos acumulados como fuentes de financiamiento a corto plazo para financiar sus operaciones $(2,00)$, utilizan los sueldos o salarios para financiar sus operaciones como parte de los pasivos acumulados $(1,58)$, y si utilizan los impuestos para financiar sus operaciones dentro de los pasivos acumulados como fuente de financiamiento a corto plazo $(2,08)$; mostrando todas estas actividades como debilidades del sector hotelero en el municipio Cabimas, en cuanto al manejo de sus estrategias de financiamiento, de acuerdo a lo establecido en el baremo de interpretación de la media.

En lo concerniente al indicador crédito comercial se observa, en la tabla referida, una media de 3,39, con moderada aplicación, siendo una debilidad para este sector; esto es generado por una moderada aplicación de los ítems medidos, donde moderadamente aplican el crédito comercial como fuente de financiamiento a corto plazo para sus operaciones (3,33), evalúan las condiciones del crédito comercial así como su ciclo de conversión en efectivo $(3,50)$, y consideren rentable utilizar el crédito comercial como fuente de financiamiento a corto plazo (3,33); lo cual convierte estas actividades en debilidades para el sector hotelero en la aplicación del crédito comercial como fuente de financiamiento interna a corto plazo.

Por último, para el indicador cuentas por pagar, catalogado con baja aplicación $(2,58)$, se observa, moderada aplicación en cuanto a si son utilizadas las cuentas por cobrar como garantía para financiamiento a corto plazo $(2,75) ;$ y con baja aplicación el que sean evaluados los costos de la cesión de la cuentas por cobrar como garantía para el financiamiento a corto plazo $(2,50)$ y utilicen el factoraje de las cuentas por cobrar para su financiamiento a corto plazo (2,50); delineando estas tres (3) actividades como debilidades del sector en el manejo de sus cuentas por pagar como fuente interna de financiamiento a corto plazo. 
Los resultados muestran, el financiamiento interno en los hoteles estudiados se basa en la aplicación del financiamiento de los socios, no obstante, moderadamente aplican el crédito comercial, pero dan baja aplicación a los pasivos acumulados y a las cuentas por pagar, constituyendo así la manera en la cual éstos captan los fondos o recursos financieros para llevar a cabo sus metas de crecimiento y progreso, siendo su estructura de financiamiento interna a corto plazo. De esta forma, pudiera entenderse como una fuente muy ventajosa para la empresa debido a su bajo costo, pues estos fondos provienen de sus socios, pero no asumiendo las otras fuentes existentes.

En este recorrido del análisis, se presentan a continuación los resultados obtenidos para cada uno de los indicadores que conforman la segunda sub-dimensión, nombrada como fuentes externas a corto plazo. Se acota, los indicadores de esta sub-dimensión están constituidos por las líneas de crédito y los proveedores. El resumen de resultados para este punto se recoge en la tabla 4.

Como puede observarse en la tabla reseñada, en las fuentes externas a corto plazo el sector hotelero estudiado muestra mayor aplicación de la fuente proporcionada por los proveedores que por la línea de crédito, al arribar la primera a muy alta aplicación $(4,25)$ y la segunda a alta aplicación $(3,42)$; aun cuando ambas son consideradas como fortalezas de sus estrategias de financiamiento a corto plazo.

Tabla 4. Sub-Dimensión: Fuentes externas a corto plazo

\begin{tabular}{lcl}
\hline INDICADORES & MEDIA & CATEGORÍA \\
\hline Línea de crédito & 3,42 & Alta aplicación/fortaleza \\
Proveedores & 4,25 & Muy alta aplicación/fortaleza \\
SUB - DIMENSIÓN & 3,83 & Alta aplicación/fortaleza \\
\hline
\end{tabular}

Fuente: Elaboración propia (2020)

Estos resultados, demuestran congruencia con Morales y Morales (2014), para quienes las fuentes externas a corto plazo proporcionan recursos provenientes de personas, empresas o instituciones ajenas a la organización financiada, ésta última al recibir recursos externos, contrata un pasivo el cual genera un costo financiero denominado interés y que tendrá que ser liquidado (interés y capital recibido) en los términos del contrato de préstamo que ampara la transacción.

Asimismo, se logra coincidencia con el investigador cuando asevera, las fuentes externas de financiamiento a corto plazo constituyen un recurso indispensable para la empresa en momentos donde requiere realizar inversiones o carece de liquidez para enfrentar los compromisos provenientes del capital de trabajo, por tanto utiliza para ello la línea de crédito y los proveedores, de acuerdo al sector que se analiza.

Al desglosar la sub-dimensión, a través de los indicadores e ítems utilizados para medirla, se observa muy alta aplicación de los proveedores como fuente externa a corto plazo $(4,25)$, siendo una fortaleza para el sector hotelero en cuanto a sus estrategias de financiamiento a corto plazo. Se puede evidenciar también como otorgan muy alta aplicación al que los proveedores le establecen 
condiciones de pago $(4,25)$, y si han pagado, en los términos acordados, el crédito comercial otorgado por los proveedores (4,33). A su vez conceden alta aplicación al que financien a corto plazo con sus proveedores $(4,17)$, convirtiendo todas las actividades medidas en estos ítems en fortalezas de sus estrategias de financiamiento externas a corto plazo.

En cuanto al indicador línea de crédito, al cual califican con alta aplicación $(3,42)$, se puede decir que estos hoteles aplican en alta medida la línea de crédito para contribuir al desarrollo del capital $(3,83)$ catalogada como una fortaleza de sus estrategias de financiamiento externas a corto plazo. Mientras, conceden moderada aplicación a la utilización de la línea de crédito como fuente de financiamiento a corto plazo $(3,25)$, y a si los intereses pagados por la línea de crédito son a tasas de interés flotantes $(3,17)$, siendo debilidades de estas estrategias, según lo establece el baremo utilizado.

Lo observado valida a Mora (2017) quien afirma, cuando una empresa periódicamente necesita fondos para diversos propósitos es probable le resulte útil negociar una línea de crédito con su banco. Asimismo, valida a Oldenburg y otros (2014) cuando afirma, el financiamiento de proveedores es el más importante debido a la ausencia de costos, el alto monto de los créditos recibidos de todos los proveedores, revolvencia automática y el incremento casi automático del monto a financiar.

De igual forma, es congruente con el investigador cuando establece, la línea de crédito constituye fuente y herramienta de financiamiento de gran utilidad y fácil consecución, la cual le facilita a los hoteles sometidos a estudio, disponer de una cantidad de dinero para realizar transacciones de bajo monto hasta el límite determinado por el banco, guiándose por la solvencia de la empresa y sus necesidades crediticias. En cuanto a los proveedores, es la más común y frecuentemente utilizada, pues la misma se genera en la adquisición o compra de bienes o servicios que la empresa utiliza en su operación a corto plazo, lo cual es aplicable al sector hotelero del municipio Cabimas.

\section{CONCLUSIONES}

En relación al objetivo, donde se identificaron las estrategias de financiamiento a corto plazo aplicadas por el sector hotelero del municipio Cabimas, se pudo evidenciar que estos hoteles poseen moderada aplicación de estas estrategias en su proceso de financiamiento, delineándola como debilidades. En específico, se conoció moderada aplicación de todas las estrategias a corto plazo analizadas, tanto de fuentes internas como externas, señalándolas como debilidades de este sector en la aplicación de sus estrategias de financiamiento.

En relación a las fuentes internas, solo el financiamiento a los socios se aplica de forma alta, mientras el resto (pasivos acumulados, crédito comercial y cuentas por pagar) se aplican de forma moderada. En lo concerniente a las fuentes externas, se evidenció que estos hoteles poseen adecuados niveles de aplicación de estas fuentes, tanto en el financiamiento de los proveedores como de la línea de crédito.

No obstante, se acota que solo en el caso de la fuente externa proveedores no se ameritan acciones estratégicas, pues todas sus actividades relacionadas se posicionan en muy alta y alta aplicación, pero el resto de los indicadores de ambas fuentes (interna y externa) muestran oportunidades de mejora y éstas se abordaron en las acciones estratégicas establecidas. 


\section{REFERENCIAS}

Chillida, C. (2005) Análisis e interpretación de balances. Ediciones de la Biblioteca de la Universidad Central de Venezuela (II edición). Caracas. Venezuela.

García, J. y Paredes, L. (2014). Estrategias Financieras Empresariales. Grupo Editorial Patria. México.

Gómez, J. (2013). Dirección Financiera I. Editorial Club Universitario. Alicante. España.

Gutiérrez, O. (2010) Análisis del entorno macroeconómico y de planificación estratégica Tercera edición. Caracas. Venezuela

Mora, C. (2017). Las fuentes de financiamiento a corto plazo como estrategia para el incremento de la productividad empresarial en las Pymes. Revista
Científica Dominio de las Ciencias. Vol. 3, $\mathrm{N}^{\circ}$ Especial, marzo, 2017, pp. 338-351. (En línea) Disponible en: http://dominiodelasciencias.com/ojs/index. php/es/index

Morales, A. y Morales, J. (2014). Planeación Financiera. Ediciones ECOE. Colombia.

Oldenburg, A.; Belloso, L.; Loaiza, C. y Alaña, W. (2014). Niveles de endeudamiento a corto plazo de las Pymes del municipio Miranda. X Jornadas Investigación y Postgrado. Facultad de Ciencias Económicas y Sociales. Universidad del Zulia. Venezuela

Sánchez, J. (2013). Indicadores de gestión empresarial: de la estrategia a resultados. Editorial Palibrio. Estados Unidos de América 